\title{
Identification of ventrolateral intramedullary intervertebral disc herniation in a dog
}

\author{
Authors: \\ Masato Kitagawa ${ }^{1}$ \\ Midori Okada² \\ Kiichi Kanayama ${ }^{1}$ \\ Takeo Sakai ${ }^{1}$ \\ Affiliations: \\ ${ }^{1}$ Department of \\ Veterinary Medicine, \\ Nihon University College \\ of Bioresource Sciences, \\ Japan \\ ${ }^{2}$ Pet Clinic ANIHOS, \\ Itabashiku, Japan

\section{Correspondence to:} \\ Masato Kitagawa \\ Email: \\ kitagawa@brs.nihon-u.ac.jp \\ Postal address: \\ 1866 Kameino, Fujisawa, \\ Kanagawa 252-0880, Japan \\ Dates: \\ Received: 02 Feb. 2011 \\ Accepted: 10 Apr. 2012 \\ Published: 07 Sept. 2012 \\ How to cite this article: \\ Kitagawa, M., Okada, M., \\ Kanayama, K. \& Sakai, T. \\ 2012, 'Identification of \\ ventrolateral intramedullary \\ intervertebral disc herniation \\ in a dog', Journal of the \\ South African Veterinary \\ Association 83(1), Art. \#103, \\ 4 pages. http://dx.doi. \\ org/10.4102/jsava.v83i1.103
}

(C) 2012. The Authors. Licensee: AOSIS OpenJournals. This work is licensed under the Creative Commons Attribution License.
A 10-year-old male cross-breed dog was brought to Nihon University Animal Hospital with a history of acute onset of paralysis in the pelvic limbs 13 days previously. Magnetic resonance imaging revealed an intramedullary linear tract in the spinal cord at the thoracic vertebrae 12-13 region, which appeared hyperintense on T2-weighted images, but was hypointense and isointense on T1-weighted images when compared with normal parenchyma of the spinal cord. A hemilaminectomy was performed and a blob of what appeared to be fibrous tissue was found adhering to the surface of the dura mater. The diameter of the blob was about $4 \mathrm{~mm}$. A durotomy was performed over the affected area and chondroid material was found within the spinal cord. Material from the nucleus pulposus penetrated the dura mater from the ventral aspect of the spinal cord in previously reported intramedullary intervertebral disc herniation cases, but, in this case, penetration occurred from the left ventrolateral aspect and progressed through to the right lateral aspect, forming a visible blob of what appeared to be fibrous tissue on the surface of the dura mater at the exit point. To the best of our knowledge, this was the first case report of an intramedullary intervertebral disc herniation originating from the ventrolateral aspect of the spinal cord in a dog.

\section{Introduction}

Intramedullary intervertebral disc herniation (IMIDH) is a rare intervertebral disc disorder where intervertebral disc material enters the spinal cord by penetrating the dura mater (Liptak et al. 2002; McConnell \& Garosi 2004; Sanders, Bagley \& Gavin 2002). Magnetic resonance imaging (MRI) is useful for diagnosing intramedullary disorders (McConnell \& Garosi 2004; Sanders et al. 2002). A number of studies have reported MRI findings of intradural disc herniation in humans (D'Andrea et al. 2004). However, only a few studies have examined them in the veterinary field (Liptak et al. 2002; McConnell \& Garosi 2004; Sanders et al. 2002). In this article, we report on the MRI findings of IMIDH in a dog.

\section{Case history}

A 10-year-old male cross-breed dog was brought to Nihon University Animal Hospital with a history of an acute onset of pelvic limb paralysis 13 days previously. At initial presentation, deep pain sensation was noted to be absent in both pelvic limbs and methylprednisolone sodium succinate $(30.0 \mathrm{mg} / \mathrm{kg}$ and $5.4 \mathrm{mg} / \mathrm{kg} / \mathrm{h}$ for $24 \mathrm{~h}$ ) had been administered intravenously (IV) at a local animal clinic. Clinical examination revealed the dog's body weight to be $11.0 \mathrm{~kg}$, body temperature $39.8^{\circ} \mathrm{C}$, heart rate 160 beats per min, and the presence of panting. Cardiac murmurs (Grade 3 in the Levine grade of cardiac murmurs) were detected and mitral regurgitation was noted on ultrasonography. Routine blood tests were performed and indicated a stress leucogram, otherwise all findings were within normal limits. On neurological examination, proprioception and postural responses, such as placing and hopping reflexes, were absent and the patellar and cranial tibial reflexes were exaggerated in both pelvic limbs. Deep pain sensation was absent in the right pelvic limb and almost absent in the left pelvic limb. Postural responses, extensor carpi radialis muscle reflex and flexor reflexes of the thoracic limbs were within normal limits, as were the findings from the cranial nerve examination. Urinary and faecal incontinence were also observed.

Radiological examination revealed narrowing of the thoracic vertebrae T12-T13 intervertebral space. Based on a tentative diagnosis of spinal disease involving the T3-L3 areas, the authors performed MRI and cerebrospinal fluid analysis. At the Nihon University Animal Hospital, MRI is performed routinely on dogs with suspected intervertebral disc disease because of the lower rate of complications reported with MRI and the superiority of MRI for diagnosis of intervertebral disc disease compared with myelography and computed tomography (Robertson \& Thrall 2011). Magnetic resonance imaging (FlexArt 1.5-Tesla magnetic resonance scanner; Toshiba, Tokyo, Japan) revealed an abnormal area in the spinal cord at the T12-T13 region. In addition, the 
shape of spinal cord observed was not normal (Figure 1a). A T-shaped lesion consisting of three parts was noted on the transverse MR images (Figure 1), with an upper area immediately beneath the bone and epidural tissue, an area below the top area and a linear tract. The linear tract traversed from the left ventrolateral side to the right dorsolateral side of the spinal cord, where it terminated in the T shape beneath the bone and the epidural tissue. The uppermost area was hyperintense on the T2-weighted image (time of repetition $[\mathrm{TR}]=4850 \mathrm{~ms}$, time of echo $[\mathrm{TE}]=105 \mathrm{~ms}$ ), isointense on the T1-weighted image $(\mathrm{TR}=365 \mathrm{~ms}, \mathrm{TE}=15 \mathrm{~ms})$ and enhanced by contrast medium (Figure 1a, Figure 1b and Figure 1c). The area below the uppermost area and the linear tract were hyperintense on the T2-weighted image, heterogeneously isointense and hypointense on the T1-weighted image and not enhanced by contrast medium (Figure 1a, Figure $1 \mathrm{~b}$ and Figure 1c). The spinal cord around the linear tract appeared isointense on the T1-weighted and T2-weighted transverse images (Figure 1a and Figure 1b). The epidural tissue of the right dorsolateral side also appeared hyperintense on the T2-weighted image and isointense on the T1-weighted image (Figure 1a and Figure 1b). At the left dorsolateral side of the spinal cord, the epidural tissue appeared hyperintense on the T1-weighted and T2-weighted images, indicating the normal epidural fat (Figure 1a and Figure 1b).

On the T1 $(\mathrm{TR}=365 \mathrm{~ms}, \mathrm{TE}=15 \mathrm{~ms})$ and $\mathrm{T} 2(\mathrm{TR}=4050 \mathrm{~ms}$, $\mathrm{TE}=105 \mathrm{~ms}$ ) sagittal image, a dorsal line on the spinal cord, which indicated fat, was lost (the linear tract did not appear on the T1 and T2 transverse images) (Figure 2a and Figure 2b). At the T12-T13 intervertebral space, a narrowed intervertebral space, decreased MRI intensity and loss of the disc were observed on the T2-weighted sagittal image. These findings suggest that the lesion was an intra-axial lesion.

Differential diagnoses of intra-axial lesions include tumour, inflammatory disease, infarction and haemorrhage, amongst others. Contrast T1-weighted imaging was performed for differential diagnosis (Gavin, Bagley \& Holmes 2009). Contrast T1-weighted transverse section $(\mathrm{TR}=410 \mathrm{~ms}$, $\mathrm{TE}=15 \mathrm{~ms}$ ) (Prohance ${ }^{\circledR}$ [gadoteridol]; Eizai, Tokyo, Japan; $0.3 \mathrm{mg} / \mathrm{kg}$, IV) revealed enhancement of the uppermost area and the intramedullary area around the T-shaped lesion on the left side of the spinal cord, but did not enhance the lower area and the linear tract (Figure 1c). The protein level, cell count and Pandy's test findings for the cerebrospinal fluid analysis were within the normal ranges.

Despite being informed of the poor surgical prognosis (deep pain loss for $>24 \mathrm{~h}$ ) (Brisson 2010), the dog's owner elected to have surgery performed. After the hemilaminectomy, a blob of what appeared to be fibrous tissue was noted adhering to the dura mater on the right dorsolateral spinal cord. Once this material was removed, a small hole was seen to penetrate through the dura mater and material that appeared to be cartilaginous was noted to be present within the spinal cord (Figure 3a and Figure 3b). The hole in the dura mater was enlarged and the cartilaginous substance was carefully removed (Figure 3c). A purple-coloured area was observed on the left side of the spinal cord. After placement of absorbable gelatin sponge, the dura mater was left unsutured (Sharp \& Wheeler 2005). The muscle and skin were closed routinely.

Pathological examination diagnosed the intramedullary cartilaginous material as the nucleus pulposus (NP). On neurological examination two weeks after the surgery, the left pelvic limb regained voluntary function, but the postural reactions, such as proprioception and hopping, were reduced. The right pelvic limb remained paralysed without deep pain sensation. Urinary and faecal incontinence persisted. Fifty days after surgery, the dog was able to stand and walk on three legs, but sometimes fell down on a slippery floor. Three months after surgery, the postural reactions of the right limb remained reduced, but the dog became to be able to walk. Faecal and urinary continence had improved slightly, but control was still poor.

\section{Ethical considerations}

We performed the MRI and surgery for the dog after we gave informed consent and received the owner's agreement.

\section{Discussion}

In the veterinary field, few studies have been reported involving IMIDH. In addition, few studies have presented MRI findings of intra-axial disc herniation (Liptak et al.
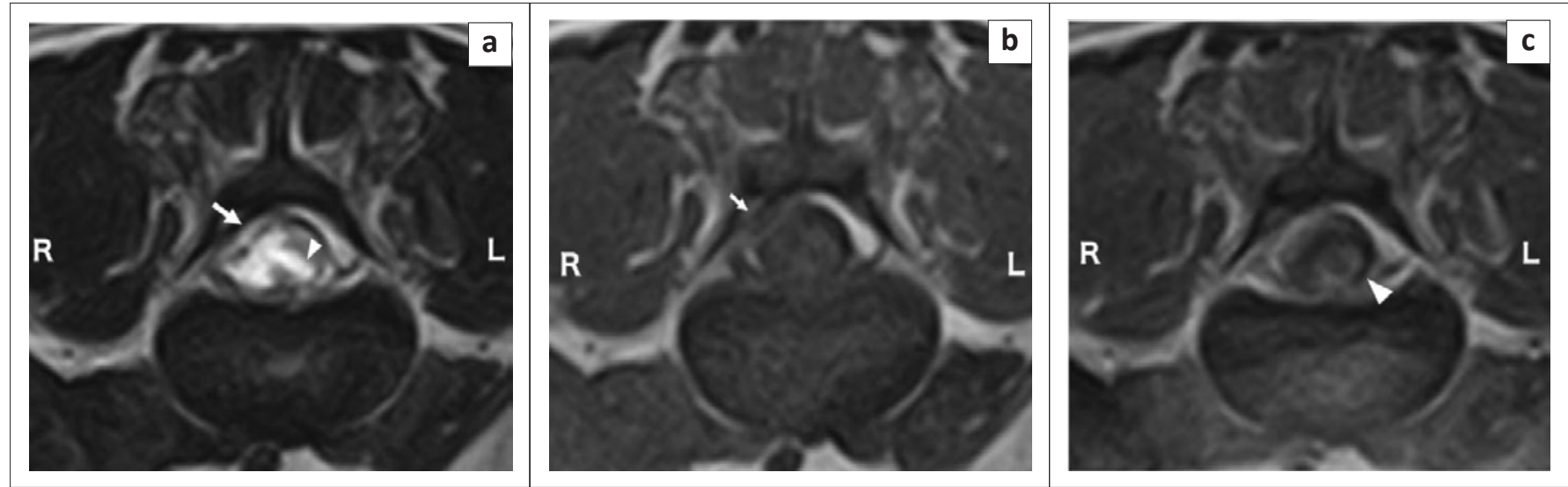

FIGURE 1: Transverse sections of the T12-T13 space, (a) T2-weighted image showing hyperintense T-shaped lesion (arrowhead), noting that both dorsolateral sides appear hyperintense (arrow), (b) T1-weighted image showing heterogeneously mixed hypointensity and isointensity of the lower area and the linear tract, noting that the top area of the T-shaped lesion appeared isointense, whilst the right dorsolateral side appears isointense (small arrow) and (c) a contrast T1-weighted image showing enhancement of the spinal cord beside the T-shaped area (arrowhead). 

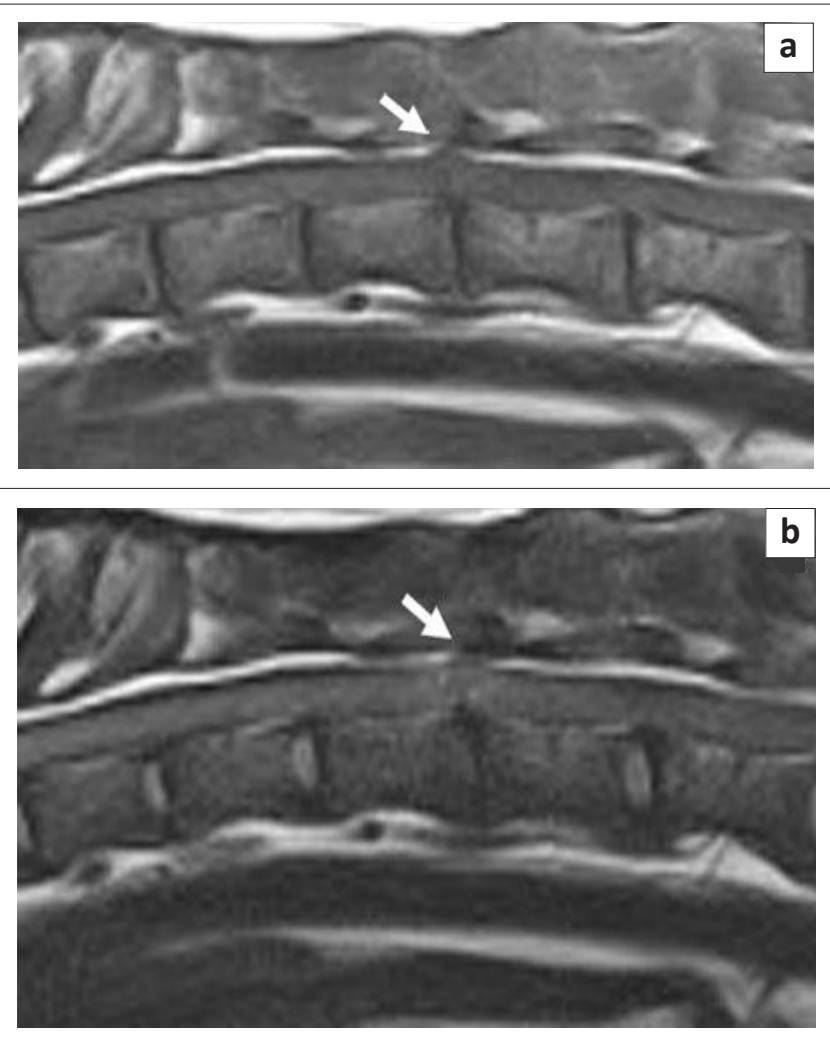

FIGURE 2: Degeneration of the intervertebral disc between the T12 and T13 vertebrae is observed in the (a) T1-weighted sagittal section and (b) T2 weighted sagittal section. The hyperintense line indicative of the epidural fat has disappeared on the dorsal side of the spinal cord (arrow).

2002; McConnell \& Garosi 2004; Sanders et al. 2002). In these reports, abnormal MRI findings were spinal swelling and the presence of hyperintense vertical and linear tracts involving the intervertebral disc space on T2-weighted MRI (McConnell \& Garosi 2004; Sanders et al. 2002). Linear tracts and focal hypointense areas on the T2 and T1 images are reported to be important for the diagnosis of IMIDH (McConnell \& Garosi 2004). Chondroid tissue of a degenerative NP appears hypointense on T1-weighted and T2-weighted images as a result of the degenerative loss of water from the disc (Zhou et al. 2007). In the present case, a linear tract was seen which was hyperintense on the T2-weighted images and was heterogeneously mixed isointense and hypointense on the T1-weighted images. The top area of the T-shaped lesion on the right dorsolateral side was hyperintense on the T2weighted images and isointense on the T1-weighted images and was enhanced with contrast medium. The shape of the spinal cord was also altered on the transverse images (Figure 1a), with the cord being compressed or displaced and swelling as a result of oedema and inflammation. Also, the blob adhering the spinal cord was noted intraoperatively. The top area of the T-shaped lesion on the right side of the spinal cord was considered to be the blob adhering to the dura mater. Focal hypointensity was not found on the T2-weighted image, although the degenerative NP was found during surgery. It was suspected that oedema of the spinal cord masked the signals of the NP. Also, the NP might include some degree of water, which influenced the signal (Gavin et al. 2009). It is speculated that the degenerated NP penetrated from the left ventrolateral side of the spinal cord and went through the spinal cord to the right side, thereby creating the linear tract. Moreover, it is speculated that the dura mater was torn by disc material and an extradural connective tissue blob was formed at this exit site.

In one of the two cases reported previously, the periphery of the linear tract and the meninges were enhanced by contrast medium (McConnell \& Garosi 2004; Sanders et al. 2002). The periphery of the linear tract was similarly enhanced in this case. Marked discolouration was observed on the left side of the spinal cord intraoperatively, which may have been caused by haemorrhage and/or inflammation after the initial incident. Haemorrhage and inflammation may cause enhancement on MRI and could explain the peripheral enhancement seen in these cases (Brisson 2010). The hyperintense line seen dorsal to the spinal cord on T1 and $\mathrm{T} 2$ sagittal images was attenuated at the T12-T13 site, which suggested that the spinal cord was displaced or had become swollen, thereby causing compression of the extradural fat. It appeared that the spinal cord had changed shape on the MRI transverse images (Figure 1a). The change in shape of the spinal cord is considered to be caused by the blob adhering to the dura mater.
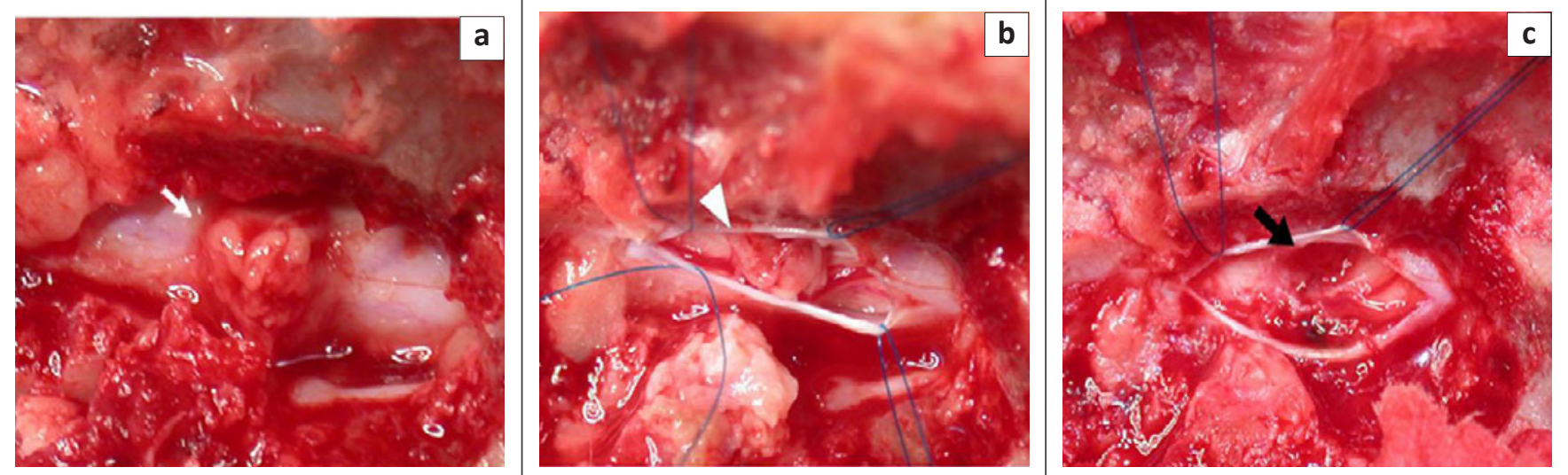

FIGURE 3: Intraoperative photographs of (a) spinal cord after hemilaminectomy, showing a fibrous-appearing blob adhering to the dura mater (arrow), (b) the spinal cord after incision of the dura mater, with a chondroid substance seen to be penetrating the spinal cord intramedullarly (arrowhead) and (c) the spinal cord after the removal of the chondroid substance. Note the spinal cord discolouration and deformity (black arrow). 
The mechanism of intradural disc herniation in humans may involve dural adhesion, congenital weakness and rapid compression of the intervertebral discs (Suzer, Tahta \& Coskun 1997). A case in which the ventral dura mater adhered to the dorsal longitudinal ligament has also been reported (Suzer et al. 1997). Two previous cases reported an abnormal signal intensity involving the intervertebral disc space on MRI (McConnell \& Garosi 2004; Sanders et al. 2002). In one of these two cases, intramedullary material was diagnosed as mature cartilage which was indistinguishable from NP histopathologically (Sanders et al. 2002). In the case reported here, it is speculated that an extruded intervertebral disc penetrated the dura mater initially on the left ventorolateral side and then penetrated the dura mater again on the right side. A blob of fibrous-like tissue was then formed in the epidural space during the process of repair. It should also be noted that whilst the previous cases reported were examined within three days of onset (Liptak et al. 2002; Sanders et al. 2002), the present case was examined 13 days after onset and this may explain the extradural blob of fibrous-like tissue.

\section{Conclusion}

A case of canine ventrolateral IMIDH at T12-T13 was reported that was diagnosed using MRI and what appeared to be prolapsed NP was removed during surgery. Although the dog was paraplegic without deep pain in either of the pelvic limbs before surgery, it became able to walk three months after surgery but had ongoing significant neurological problems. This report is, as far as could be ascertained, the first report of ventrolateral intramedullary intervertebral disc herniation in a dog.

\section{Acknowledgements}

The authors would like to thank the NORTH LaB ${ }^{\circledR}$ for performing the pathological examination.

\section{Competing interests}

The authors declare that they have no financial or personal relationship(s) which may have inappropriately influenced them in writing this paper.

\section{Authors' contributions}

M.K. (Nihon University College of Bioresource Sciences) performed most of the clinical examinations and surgery. M.K. (Pet Clinic ANIHOS) and M.O. (Pet Clinic ANIHOS) interpreted the MRI. M.K. (Nihon University College of Bioresource Sciences), T.S. (Nihon University College of Bioresource Sciences) and K.K. (Nihon University College of Bioresource Sciences) wrote the manuscript.

\section{References}

Brisson, B.A., 2010, 'Intervertebral disc disease in dogs', Veterinary Clinics of North America Small Animal Practice 40, 829-858. http://dx.doi.org/10.1016/j. cvsm.2010.06.001, PMid:20732594

D’Andrea, G., Trillò, G., Roperto, R., Celli, P., Orlando, E.R. \& Ferrante, L., 2004, 'Intradural lumbar disc herniations: The role of MRI in preoperative diagnosis and review of the literature', Neurosurgical Review 27, 75-80. http://dx.doi org/10.1007/s10143-003-0296-3, PMid:14564663

Gavin, P.R., Bagley, R.S. \& Holmes, S.P., 2009, 'Diagnosis of spinal disease', in P.R. Gavin \& R.S. Bagley (eds.), Practical small animal MRI, pp. 123-226, Wiley-Blackwell, Ames. http://dx.doi.org/10.1002/9780813810324

Liptak, J.M., Allan, G.S., Krockenberger, M.B., Davis, P.E. \& Malik, R., 2002, 'Radiographic diagnosis: Intramedullary extrusion of an intervertebral disc', Veterinary Radiology and Ultrasound 43, 272-274. http://dx.doi.org/10.1111/j.1740-8261.2002.tb01002.x

McConnell, J.F. \& Garosi, L.S., 2004, 'Intramedullary intervertebral disk extrusion in a cat', Veterinary Radiology and Ultrasound 45, 327-330. http://dx.doi. org/10.1111/j.1740-8261.2004.04062.x

Robertson, I. \& Thrall, D.E., 2011, 'Imaging dogs with suspected disc herniation: Pros and cons of myelography, computed tomography, and magnetic resonance' Veterinary Radiology and Ultrasound 52, S81-s84. http://dx.doi.org/10.1111/ j.1740-8261.2010.01788.x

Sanders, S.G., Bagley, R.S. \& Gavin, P.R., 2002, 'Intramedullary spinal cord damage associated with intervertebral disk material in a dog', Journal of the American Veterinary Medical Association 221, 1594-1596. http://dx.doi.org/10.2460/ javma.2002.221.1594, PMid:12479331

Sharp, N.J.H. \& Wheeler, S.J., 2005, Small animal spinal disorder diagnosis and surgery, 2nd edn., Elsevier Mosby, Edinburgh.

Suzer, T., Tahta, K. \& Coskun, E., 1997, 'Intraradicular lumbar disc herniation: Case report and review of the literature', Neurosurgery 41, 956-958. http://dx.doi. org/10.1097/00006123-199710000-00037

Zhou, H., Hou, S., Shang, W., Wu, W., Cheng, Y., Mei, F. et al., 2007, 'A new in vivo animal model to create intervertebral disc degeneration characterized by MRI, radiography, CT/discogram, biochemistry, and histology', Spine 15, 864-872. http://dx.doi.org/10.1097/01.brs.0000259835.31062.3d, PMid:17426630 